\title{
Association between poor oral health and eating disorders: systematic review and meta-analysis
}

\author{
Steve Kisely, Hooman Baghaie, Ratilal Lalloo and Newell W. Johnson
}

\section{Background}

There is a well-established link between oral pathology and eating disorders in the presence of self-induced vomiting.

There is less information concerning this relationship in the absence of self-induced vomiting, in spite of risk factors such as psychotropic-induced dry mouth, nutritional deficiency or acidic diet.

\section{Aims}

To determine the association between eating disorder and poor oral health, including any difference between patients with and without self-induced vomiting.

\section{Method}

A systematic search was made of Medline, PsycINFO, EMBASE and article bibliographies. Outcomes were dental erosion, salivary gland function and the mean number of decayed, missing and filled teeth or surfaces (DMFT/S).

\section{Results}

Ten studies had sufficient data for a random effects metaanalysis (psychiatric patients $n=556$, controls $n=556$ ).
Patients with an eating disorder had five times the odds of dental erosion compared with controls $(95 \% \mathrm{Cl} 3.31-7.58)$; odds were highest in those with self-induced vomiting (odds ratio $(\mathrm{OR})=7.32$ ). Patients also had significantly higher DMFS scores (mean difference 3.07, 95\% $\mathrm{Cl} 0.66-5.48$ ) and reduced salivary flow $(\mathrm{OR}=2.24,95 \% \mathrm{Cl} 1.44-3.51)$.

\section{Conclusions}

These findings highlight the importance of collaboration between dental and medical practitioners. Dentists may be the first clinicians to suspect an eating disorder given patients' reluctance to present for psychiatric treatment, whereas mental health clinicians should be aware of the oral consequences of inappropriate diet, psychotropic medication and self-induced vomiting.

\section{Declaration of interest} None.

\section{Copyright and usage}

(c) The Royal College of Psychiatrists 2015.
The complex interrelationships between physical and psychiatric illnesses have been the focus of much research. ${ }^{1}$ Depression, for example, is not only a serious chronic illness but also a major risk factor for heart disease and cancer. At the same time there is strong evidence that mental health is important for maintaining good physical health and healthy lifestyle practices. However, the relationship between oral and mental health is a relatively neglected area. In the only meta-analysis of the association between the two, patients with psychiatric disorders such as schizophrenia, bipolar disorder and dementia were over three times as likely to have lost all their teeth. ${ }^{2}$ However, this study did not include eating disorders.

Eating disorders are divided into three main diagnoses. ${ }^{3}$ Anorexia nervosa is characterised by low body weight and food restriction. Bulimia nervosa is characterised by binge eating and inappropriate compensatory behaviours such as self-induced vomiting, use of laxatives and excessive exercise. Eating disorder not otherwise specified includes a mixture of anorexia- and bulimia-like atypical disorders. ${ }^{3}$ The impact of eating disorders on oral health was initially reported by Hellstrom and Hurst et al in the late 1970s. ${ }^{4,5}$ There are three main types of oral pathology. ${ }^{3,6-9}$ Dental erosion or pathological wear on tooth surfaces is defined as loss of dental tissue without the involvement of bacteria; $^{8}$ risk factors include the consumption of large amounts of citrus fruit, soft drinks and sports drinks, as well as the presence of gastric reflux or frequent vomiting. In contrast, dental caries is the result of bacterial action; ${ }^{8}$ organic acids produced by microorganisms in dental plaque cause decalcification of the tooth enamel and subsequent destruction of enamel and dentin. Finally, self-induced vomiting or starvation can lead to hyposalivation and xerostomia (dry mouth). This may be accentuated by psychotropic medication. ${ }^{8}$ Hyposalivation and xerostomia are risk factors for both dental caries and erosion.
The association between oral pathology and eating disorders is most clearly established in cases with frequent self-induced vomiting, regardless of whether the diagnosis is anorexia or bulimia, and is characterised by dental erosion on palatal surfaces (the inner surfaces of teeth in the upper jaw). ${ }^{7,8}$ Dental caries and dry mouth secondary to salivary gland dysfunction also occur. Oral pathology is less clearly established in patients with an eating disorder but without self-induced vomiting, particularly in the case of dental caries. ${ }^{7}$ On one hand, people with anorexia without self-induced vomiting might be more at risk through additional factors such as nutritional deficiency or the use of carbonated drinks as appetite suppressants; ${ }^{6}$ on the other hand, the frequent co-occurrence of obsessional personality traits might mean such people are more fastidious in their oral hygiene. ${ }^{6}$ We therefore undertook a meta-analysis to determine the association between eating disorders and poor oral health including any differences between patients with and without self-induced vomiting. Although there have been several systematic reviews, none included any meta-analysis. ${ }^{3,7,8}$

\section{Method}

The review was registered with PROSPERO, an international database of prospectively registered systematic reviews in health and social care based in the UK. ${ }^{10}$ In addition, we followed recommendations for the reporting of Meta-analyses of Observational Studies in Epidemiology (MOOSE), including background, search strategy, methods, results, discussion and conclusions. ${ }^{11}$

\section{Oral health outcomes}

The primary outcome of this study was dental erosion. This can be expressed as either a continuous or a dichotomous variable. In 
either situation, the area of the mouth that is worst affected determines the overall score. The pattern of erosion is described by the direction in which the tooth surface faces (see online Fig. DS1). In terms of the outer surfaces, buccal surfaces are adjacent to the cheeks, whereas labial ones face the lips. Inner surfaces adjacent to the tongue are called lingual; ${ }^{12}$ sometimes these surfaces are called palatal when referring to the upper teeth adjacent to the palate. The top areas are divided into the occlusal surfaces (the chewing surfaces of posterior teeth) and the incisal edges (the biting edge of anterior teeth). ${ }^{12}$

Secondary outcomes were dental decay and salivary gland function. Dental caries was assessed by the number of decayed, missing and filled teeth or surfaces. Both scores are expressed as a continuous variable that accumulates over a person's lifetime, reflecting the individual's overall experience of dental caries. ${ }^{13}$ This is because both dental decay and its treatment leave permanent marks, either through the continued presence of carious lesions, the presence of fillings or the loss of affected teeth by extraction. The total number of teeth $(\mathrm{T})$ and surfaces $(\mathrm{S})$ that are decayed (D), missing because of pathology (M) or filled (F) are measures referred to as the DMFT and DMFS respectively. In both measures an increase in score means greater cumulative dental decay; however, DMFS scores are higher than DMFT scores because the former measure counts damage to each surface of every tooth rather than counting the tooth as a single unit. This can be four or five surfaces depending on the tooth. The maximum possible DMFT score is therefore 32, whereas the maximum DMFS score is 148. Salivary gland function was assessed where possible by measurement of unstimulated salivary flow, otherwise by report of dry mouth by the patient. Both outcomes are usually reported as dichotomous variables; in the case of saliva flow, cut-off values can be either less than $0.1 \mathrm{ml} / \mathrm{min}$ or less than $0.2 \mathrm{ml} / \mathrm{min}^{14}$

\section{Inclusion and exclusion criteria}

We included studies of the oral health of people with eating disorders that included a control group of people without eating disorders, ideally matched by age, gender, socioeconomic status and education level. Psychiatric status could be determined by clinical diagnosis or diagnostic criteria. Studies of people with severe mental illness, primary alcohol or substance use disorders, intellectual disability and other psychological disorders were excluded. As our focus was on dental erosion, decay and salivary gland function, we excluded studies of other dental outcomes such as poor oral hygiene.

\section{Search strategy}

We searched Medline, PsycINFO and EMBASE from January 1951 until June 2014 using the following text, MeSH or Emtree terms as appropriate: bulimia, binge eating, eating disorder, overeating, appetite disorder, binge eating disorder, binge eating disorders, anorexia, anorexia nervosa, oral health, dental health survey, dental care, dental health services, edentulous mouth, dental caries, dental erosion, toothloss and tooth wear. Other descriptive words associated with the above MeSH terms were also used as key terms. We searched for further publications by scrutinising the reference lists of initial studies identified and other relevant review papers. We made attempts to contact selected authors and experts. Two reviewers (H.B. and S.K.) independently assessed titles, abstracts and papers, as well as extracting and checking the data for accuracy. In cases of disagreement consensus was reached on all occasions. Authors R.L. and N.W.J. provided content expertise, especially in relation to oral and dental health issues.

\section{Study quality}

We assessed the quality of included studies using the NewcastleOttawa Scale (NOS). ${ }^{15}$ This assesses the quality of non-randomised studies in meta-analyses in the three following areas:

(a) selection of the study groups in terms of case definition, representativeness (e.g. all eligible cases with the outcome of interest over a defined period of time or from a defined catchment area), source of controls (ideally the community) and checks that the controls did not have an eating disorder;

(b) comparability of the groups such as the use of matching or multivariate techniques;

(c) ascertainment of outcome such as the use of standardised or validated measures with masking to psychiatric status.

\section{Statistical analysis}

We used Review Manager version 5.0, a statistical software package for analysing a Cochrane Collaboration systematic review, for our analysis. For each outcome we divided the eating disorder group into three subgroups regardless of whether the diagnosis was anorexia or bulimia: one in which self-induced vomiting was frequent, a second in which it was absent and a third group that had a mixture or where it was not explicitly stated. Where we encountered a situation where data for the same outcome were presented in some studies as dichotomous data and in others as continuous data, we combined them using statistical approaches as recommended in the Cochrane Handbook. ${ }^{16}$ These techniques re-express odds ratios as standardised mean differences (and vice versa), allowing dichotomous and continuous data to be pooled. Once standardised mean differences (or log odds ratios) and their standard errors were computed for all studies in the meta-analysis, they were combined using the generic inverse variance method in RevMan. For dental decay and salivary flow, most studies reported dichotomous outcomes. We therefore converted continuous data to dichotomous variables and then calculated odds ratios given that the studies were a cross-sectional design. Odds ratios also have the advantage of being easier to understand and more clinically meaningful than standardised mean differences (SMD). Dental decay was consistently reported using continuous data. We calculated the mean differences (as opposed to SMD) for studies that used the same scale for each outcome (e.g. DMFT, DMFS).

We assessed heterogeneity by using the $I^{2}$ statistic. This provides an estimate of the percentage of variability due to heterogeneity rather than chance alone. An $I^{2}$ estimate of $50 \%$ or above indicates possible heterogeneity, and scores of $75-100 \%$ indicate considerable heterogeneity. ${ }^{16}$ The $I^{2}$ statistic is calculated using the chi-squared statistic $(Q)$ and its degrees of freedom. It has several advantages over the $Q$ statistic alone in that it does not depend on the number of studies in the meta-analysis and so has greater power to detect heterogeneity where the number of studies is relatively low. ${ }^{16}$ The $I^{2}$ statistic can also be interpreted similarly irrespective of whether outcome data are dichotomous or continuous.

We used a random effects model throughout as there was significant heterogeneity in the majority of our analyses. This model assumes that variations in effect among different studies are due to differences in samples or paradigms and have a normal distribution, i.e. that heterogeneity exists. In addition, where possible, we investigated heterogeneity in sensitivity analyses omitting each study in turn. Other sensitivity analyses included investigating the effects of setting (e.g. in-patient or out-patient) and of excluding studies where there were concerns about data quality. Where there were at least 10 studies we tested for 
publication bias using both the fail-safe $N$ statistic and funnel plot asymmetry. We used WinPepi version $11.34{ }^{17}$ The fail-safe $N$ statistic is the number of non-significant studies that would be necessary to reduce the odds ratio or effect size to a negligible value. In tests for a skewed funnel plot, low $P$-values suggest publication bias.

\section{Results}

We found 1085 citations of interest in the initial electronic searches, from which 112 abstracts were screened. Of these, 39 full-text papers were potentially relevant and assessed for eligibility. Assessing the references in all papers yielded one more full-text paper that was deemed relevant, giving a total of 40 full-text papers. Of these, 30 papers were excluded, most because they were not prevalence studies of oral health and eating disorder, or did not include a relevant dental outcome (Fig. 1). This left 10 studies that could be included in the meta-analysis. Four were from Nordic countries, ${ }^{18-21}$ two studies were from England, ${ }^{22,23}$ and there was one study each from Australia, ${ }^{24}$ Israel, ${ }^{25}$ Germany, ${ }^{26}$ and the USA. ${ }^{27}$ The most common diagnosis was bulimia, followed by anorexia and eating disorders not otherwise specified. Ages ranged from 10 years to 50 years. The studies are summarised in online Table DS1.

Study quality was not optimal, particularly in the areas of selection and ascertainment of outcome. Only one study stated that eating disorder cases were consecutive admissions, ${ }^{24}$ and no study gave details about participation rates. Six studies defined psychiatric caseness using diagnostic criteria such as the DSM or ICD, but this was by clinical assessment not a standardised psychiatric interview (Table DS1). One further study assessed

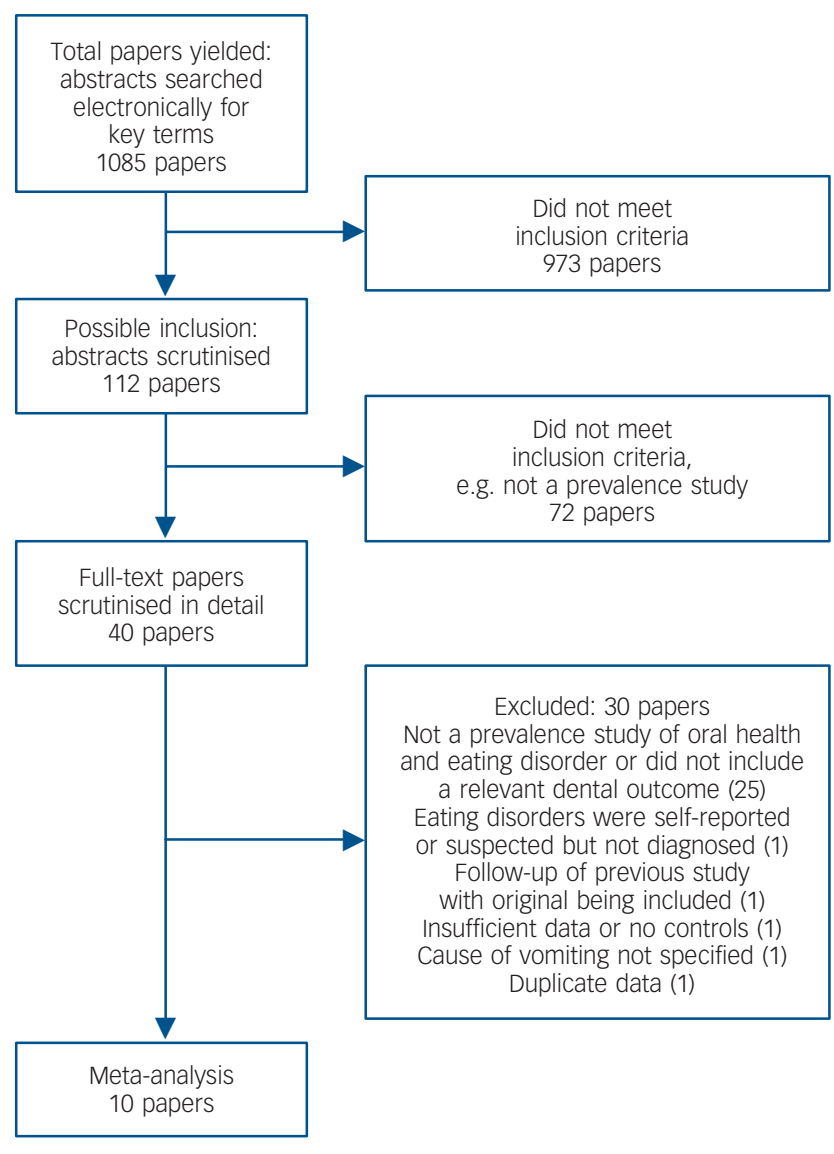

Fig. 1 Number of papers yielded by search strategy the presence of morbidity using the Eating Disorders Inventory and Examination, as well as the Eating Attitudes Test. ${ }^{24}$ None of the studies used community controls; three recruited dental patients, ${ }^{19,23,27}$ and the remainder used staff or university students. In one study the source of controls was not stated. ${ }^{26}$ One study excluded the presence of eating disorders in the control group through the use of a standardised questionnaire, ${ }^{19}$ whereas another asked about past psychiatric history. ${ }^{26}$

In terms of group comparability, all the studies either used age- and gender-matched controls or checked that there was no significant difference between the two groups at baseline. Two studies checked that participants in the eating disorder cases and control groups were of similar socioeconomic status, ${ }^{22,23}$ and a third that they were similar in terms of ethnicity and medical history. ${ }^{27}$

Ascertainment of oral status in all the studies was by trained dental examiners. In the case of erosion, this was a clinical assessment sometimes guided by an established classification. Two studies used the Tooth Wear Index. ${ }^{28}$ Five studies supplemented the clinical examination with dental impressions, radiographs and/or intraoral photographs. In the case of caries, all the studies used some or all of the Decayed, Missing and Filled classification. ${ }^{29}$ In two studies, radiographs and/or intraoral photographs were also taken. However, only one study made specific mention of assessor calibration and the measurement of interrater reliability, and another measured agreement on a random subsample of 10 participants. ${ }^{19,21}$ The dental assessor was masked to psychiatric status in four studies. ${ }^{18,19,21,22}$ Five studies assessed salivary gland function, and saliva flow was measured in all cases. (Full details are given in Table DS1.)

Data for meta-analyses were available for 556 patients with eating disorder and 556 controls (total $n=1112$ ). Gender data were available for 868 participants, of whom 852 (98\%) were female. There were two studies where a mean and range were reported. ${ }^{18,19}$ In this situation we used the range rule to estimate the standard deviation through dividing the range by four. ${ }^{30}$ However, given this is not universally accepted, ${ }^{16}$ we did a sensitivity analysis of the effect of excluding these two studies.

\section{Dental erosion}

Participants with an eating disorder had five times the odds of dental erosion (95\% CI 3.31-7.58) compared with controls (Fig. 2). Patients with self-induced vomiting had the highest likelihood (odds ratio $(\mathrm{OR})=7.32$ ) whereas those without vomiting had the lowest $(\mathrm{OR}=3.10)$, although this still remained significantly greater than for controls (95\% CI 1.67-5.77). Excluding the two studies where the s.d. was estimated from ranges made no difference to these results. The same applied when we included only studies that used diagnostic criteria to define the psychiatric cases $(\mathrm{OR}=4.95,95 \%$ CI 3.13-7.84). In terms of sensitivity analyses of the effect of setting, it was not always clear whether studies were of in-patients, day patients or out-patients. However, limiting the analyses to studies that were clearly restricted to outpatients made no difference to the results $(\mathrm{OR}=3.75,95 \% \mathrm{CI}$ 2.11-6.70). We undertook two sensitivity analyses of study quality. Including only studies that matched or checked for confounding variables such as socioeconomic status, ethnicity and medical history, ${ }^{22,23,27}$ or those where the dental assessment was made masked to psychiatric status, ${ }^{18,19,21,22}$ also did not alter the results.

\section{Dental caries}

Four studies used some or all of the DMFS classification, and a fifth used the DMFT (Fig. 3). Patients with an eating disorder 


\begin{tabular}{|c|c|c|c|c|c|c|}
\hline \multirow[b]{2}{*}{ Study or subgroup } & \multicolumn{4}{|c|}{ Eating alsorder } & \multirow{2}{*}{\multicolumn{2}{|c|}{ sht IV, random, $95 \% \mathrm{Cl}$}} \\
\hline & $\log (\mathrm{OR})$ & s.e. & Total & Total & & \\
\hline \multicolumn{7}{|l|}{ Eating disorder, no vomiting } \\
\hline Emodi-Perlman et al $(2008)^{25}$ & 1.02 & 0.64 & 36 & 24 & $5.2 \%$ & $2.77(0.79,9.72)$ \\
\hline Liew et al (1991) & 0.51 & 0.73 & 15 & 15 & $4.5 \%$ & $1.67(0.40,6.96)$ \\
\hline Milosevic \& Slade $(1989)^{22}$ & 0.73 & 0.37 & 25 & 25 & $7.6 \%$ & $2.08(1.00,4.29)$ \\
\hline Philipp et al (1991) ${ }^{26}$ & 2.86 & 0.74 & 11 & 25 & $4.5 \%$ & $17.46(4.09,74.47)$ \\
\hline Robb et al $(1995)^{23}-\mathrm{AN}$ & 1.09 & 0.36 & 54 & 54 & $7.7 \%$ & $2.97(1.47,6.02)$ \\
\hline Subtotal (95\% Cl) & & & 141 & 143 & $29.6 \%$ & $3.10(1.67,5.77)$ \\
\hline
\end{tabular}

Heterogeneity: $\tau^{2}=0.22 ; \chi^{2}=7.29$, d.f. $=4(P=0.12) ; I^{2}=45 \%$

Test for overall effect $Z=3.57(P=0.0004)$

$\begin{array}{lll}\text { Combined group } & & \\ \text { Altshuler et al (1990) })^{27} & 2.13 & 0.46 \\ \text { Johansson et al }(2012)^{19} & 2.68 & 0.4 \\ \text { Ohm et al }(1999)^{20} & 0.66 & 0.23 \\ \text { Rytomaa et al }(1998)^{21} & 1.12 & 0.2\end{array}$

Subtotal $(95 \% \mathrm{Cl})$

Heterogeneity: $\tau^{2}=0.57 ; \chi^{2}=23.41$, d.f. $=3(P<0.0001) ; I^{2}=87 \%$

Test for overall effect $Z=3.83(P=0.0001)$

\begin{tabular}{|c|c|c|}
\hline Dynesen et al (2008) ${ }^{18}$ & 2.43 & 0.6 \\
\hline Emodi-Perlman et al (2008) ${ }^{25}$ & 1.38 & 0.63 \\
\hline Milosevic \& Slade $(1989)^{22}$ & 0.93 & 0.36 \\
\hline Philipp et al (1991) $)^{26}$ & 2.37 & 0.4 \\
\hline Robb et al $(1995)^{23}-\mathrm{BN}$ & 2.49 & .53 \\
\hline Robb et al $(1995)^{23}$ - vomiting AN & 2.5 & 4 \\
\hline
\end{tabular}

Robb et al (1995) ${ }^{23}$ - vomiting AN $\quad 2.5 \quad 0.48$

Subtotal $(95 \% \mathrm{Cl})$

Heterogeneity: $\tau^{2}=0.36 ; \chi^{2}=12.84$, d.f. $=5(P=0.02) ; I^{2}=61 \%$

Test for overall effect $Z=6.24(P<0.00001)$

Total $(95 \% \mathrm{Cl})$

Heterogeneity: $\tau^{2}=0.45 ; \chi^{2}=54.87$, d.f. $=14(P<0.00001) ; I^{2}=74 \%$

556

$556 \quad 100.0 \%$

$8.41(3.42,20.73)$

$14.59(6.66,31.94)$

$1.93(1.23,3.04)$

$3.06(2.07,4.54)$

$4.84(2.16,10.84)$

Test for overall effect $Z=7.61(P=0.00001)$

Test for subgroup differences: $\chi^{2}=3.68$; d.f. $=2(P=0.16) ; I^{2}=45.3 \%$

$\begin{array}{rrrr}20 & 20 & 5.2 \% & 11.36(3.24,39.82) \\ 43 & 24 & 5.3 \% & 3.97(1.16,13.66) \\ 33 & 25 & 7.7 \% & 2.53(1.25,5.13) \\ 41 & 25 & 7.3 \% & 10.70(4.88,23.43) \\ 39 & 39 & 6.1 \% & 12.06(4.27,34.08) \\ 29 & 29 & 6.8 \% & 12.18(4.95,30.01) \\ 205 & 162 & 38.4 \% & 7.32(3.92,13.67)\end{array}$

$\mathrm{OR}$ IV, random, $95 \% \mathrm{Cl}$

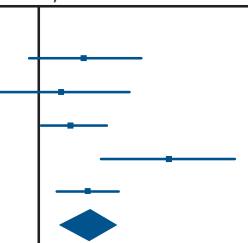

Fig. 2 Dental erosion. AN, anorexia nervosa; BN, bulimia nervosa

had significantly more decayed, missing and filled surfaces than controls. The study that used the DMFT reported no difference but this was confined to non-vomiting patients. There were insufficient studies to undertake any sensitivity analyses.

\section{Salivary gland function}

Five studies assessed salivary gland function in terms of dry mouth or reduced salivary flow and there was a significant association with eating disorders (Fig. 4). Again, there were insufficient studies to undertake any sensitivity analyses.

\section{Heterogeneity}

All but two of the results had an $I^{2}$ estimate of $50 \%$ or more, indicating possible heterogeneity. The two exceptions were erosion in the absence of self-induced vomiting and DMFS scores. There was no difference in $I^{2}$ values in sensitivity analyses of the effect of omitting each study in turn.

\section{Publication bias}

We were only able to test for publication bias for dental erosion as there were insufficient studies for the other two outcomes. The fail-safe $N$ of additional 'null' studies needed to reduce the overall odds ratio to 1.1 was 201, suggesting that the findings for erosion were reasonably robust against publication bias. Tests for funnel plot asymmetry gave a $P$-value of 0.35 (Fig. 5).

\section{Discussion}

To our knowledge, this is the first meta-analysis of the association between eating disorders and poor oral health including any difference between patients with and without self-induced vomiting. The most frequent finding was the presence of erosion or pathological wear on tooth surfaces. The risk of dental erosion is increased both in individuals who consume large amounts of citrus fruits, soft drinks or sports drinks, and in the presence of gastric reflux or vomiting. ${ }^{8}$ This is consistent with our finding that patients with self-induced vomiting have the greatest degree of oral pathology, but even in patients in whom vomiting is not a prominent symptom erosion still occurs. Dental caries and reduced salivary gland function, although less marked, remained significantly greater than in controls.

Early reports of the oral consequences of eating disorders emphasised the role of self-induced vomiting as an aetiological factor, with wear occurring especially on palatal (inner) surfaces of teeth in the upper (maxillary) arch. ${ }^{4-6,31}$ However, the relationship is not simple because the frequency, duration and total number of vomiting episodes were not linearly associated with erosion. ${ }^{6}$ In addition, later work has shown that erosion occurs in patients with an eating disorder who do not vomit, although the pattern is different with greater involvement of buccal and or labial sites (i.e. the outer surfaces of the teeth facing the cheeks and lips). ${ }^{6}$ It may therefore be simplistic to ascribe all dental effects as being secondary to vomiting when other risk factors such as acidic food and drink, and the pattern of their 


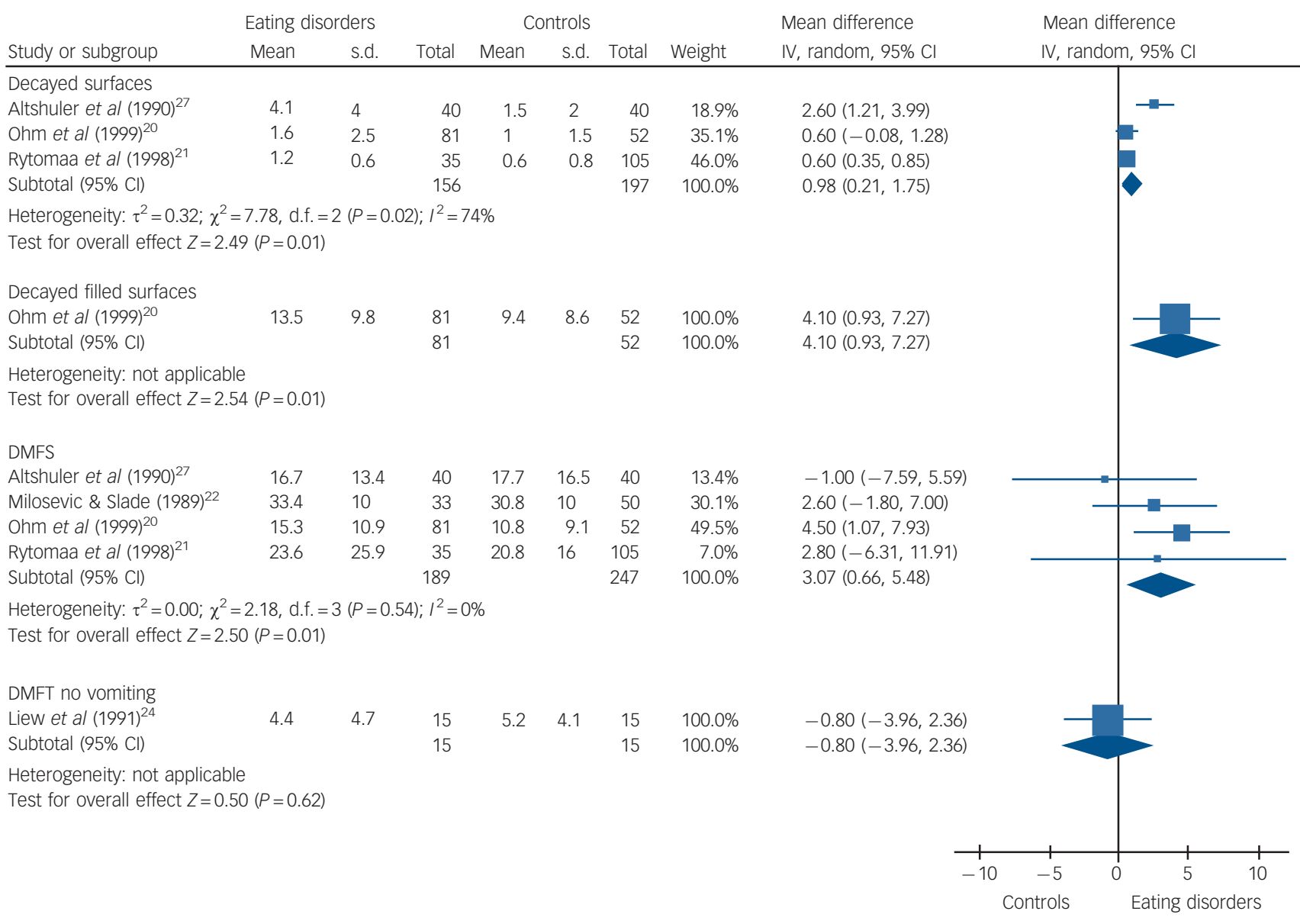

Fig. 3 Dental caries (tooth decay). DMFS/T, decayed, missing and filled surfaces/teeth.

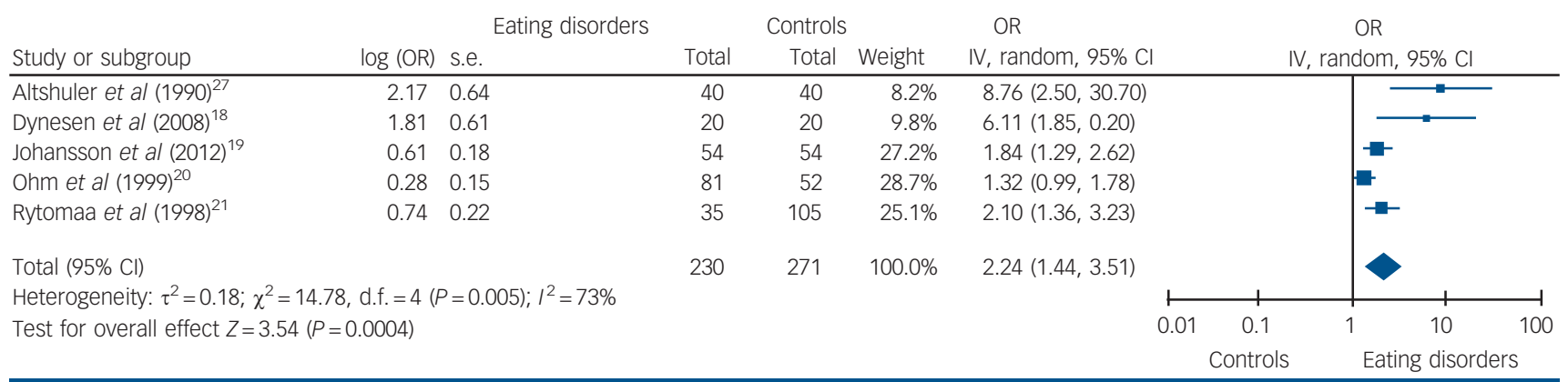

Fig. 4 Dry mouth or reduced salivary flow.

consumption, have not been studied. ${ }^{6,7}$ One theory is that intrinsic (gastric) acid results in palatal erosion, whereas extrinsic (dietary) acids from fruit or carbonated drinks lead to labial erosion. ${ }^{6}$

Dental decay and salivary gland dysfunction are closely related. Dry mouth is a major risk factor for decay, possibly compounded by an increased risk of opportunistic infections as a result of nutritional deficiencies. ${ }^{9}$ Changes in salivary secretion may be secondary to structural change within the gland, and benign parotid enlargement has been frequently described in patients with bulimia. ${ }^{6}$ Dry mouth may also be a side-effect of commonly used psychotropic medications. ${ }^{6,8}$

Poor dental health can have major consequences for patients with eating disorder. These include oral function impairment, oral discomfort or pain, poor aesthetic quality and reduced quality of life. ${ }^{9}$ In turn, deteriorating facial appearance may further alter body perception and/or self-esteem and hence contribute to a dangerous vicious cycle. ${ }^{9}$

\section{Limitations}

There are a number of limitations to our study. Study quality was not optimal. For instance, none of the studies established psychiatric caseness using the gold standard of a structured standardised interview. Although all the studies used age- and gender-matched controls, only three matched or checked for other potential confounding variables such as socioeconomic status, ethnicity and medical history. ${ }^{22,23,27}$ However, restricting the 


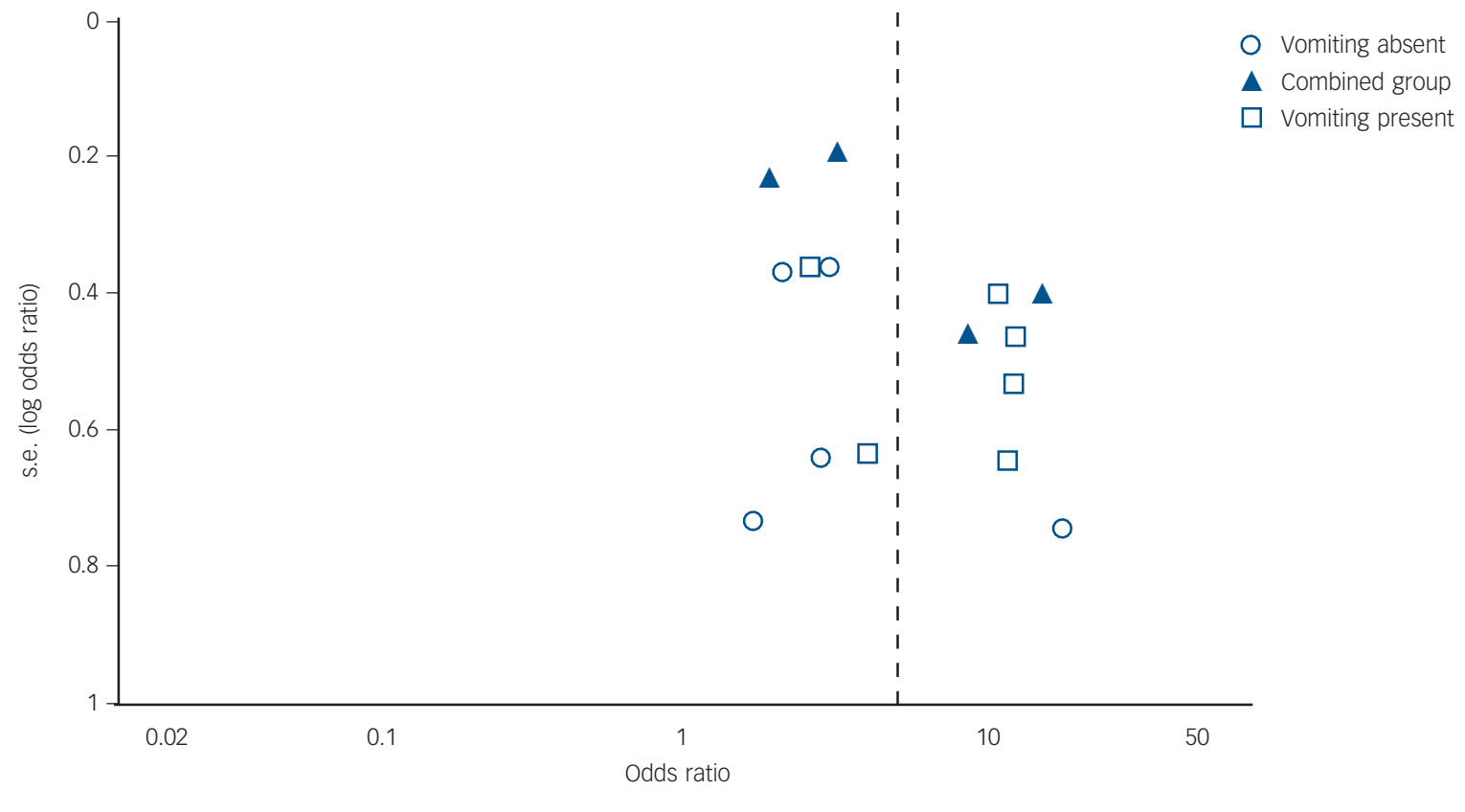

Fig. 5 Funnel plot of studies on dental erosion.

analyses to just these three studies did not change the results for our primary outcome. Although dental status in all the studies was assessed by trained examiners, in only four was this done masked to psychiatric status. ${ }^{18,19,21,22}$ Again, a sensitivity analysis of the effects of only including masked (blinded) outcomes made no difference to the erosion results. Unfortunately, there were insufficient studies for sensitivity analyses of the secondary outcomes.

There were other limitations in study quality that we could not attempt to address using sensitivity analyses, such as the calibration or standardisation of dental assessments. In addition, many of our results showed heterogeneity. We explored this further through sensitivity analyses of the effect of omitting each study in turn, but this made no difference to the results. Accordingly, we used a random effects model throughout to incorporate heterogeneity into our analyses. However, although we have tried to minimise the effects of heterogeneity, our results should still be treated with caution. Finally, we cannot exclude the possibility of publication bias even though the fail-safe $N$ for the primary outcome was 201. This is because tests for funnel plot asymmetry tend to be underpowered when the number of studies is relatively low. There were also insufficient studies to test for publication bias for the other two outcomes.

\section{Implications}

These findings highlight the importance of collaboration between dental and other health workers such as dieticians, general practitioners, psychiatrists and other mental health clinicians. This applies to all patients with eating disorders, not just those who present with self-induced vomiting. Dentists may be the first clinicians to suspect the diagnosis, given the reluctance of some people with eating disorders to present for treatment. ${ }^{9}$ In established cases collaboration might help to minimise the harmful effects of inappropriate diet and self-induced vomiting; ${ }^{6}$ for instance, patients should be advised to reduce their intake of acidic drinks and food such as citrus fruit, as well as alcohol. After episodes of self-induced vomiting they should chew gum and rinse their mouth with water, milk or an antacid preparation. ${ }^{6}$
They should brush their teeth gently with a small amount of desensitising or bicarbonate toothpaste; vigorous brushing after self-induced vomiting is inadvisable as the softened, demineralised surface is more susceptible to toothbrush abrasion. ${ }^{22}$ Finally, medical practitioners should be aware that many psychotropic medications can exacerbate dry mouth with consequent adverse effects on oral health. ${ }^{6}$ If this is unavoidable, they should prescribe neutral artificial saliva or sialogogue pastilles. ${ }^{6}$

The increased focus on physical and psychiatric comorbidity should include consideration of oral health. Policy makers should consider providing free, accessible dental care for people with eating disorders. For example, Queensland's strategy to improve the physical health of people with psychiatric illness (Activate: Mind and Body) includes both the promotion of oral hygiene and regular care from a dentist. ${ }^{32}$

Steve Kisely, MD, PhD, School of Medicine, University of Queensland Woolloongabba; Hooman Baghaie, $\mathrm{BOH}$, School of Dentistry, University of Queensland, Herston, Queensland; Ratilal Lalloo, MChD, PhD, Australian Centre for Population Oral Health, School of Dentistry, University of Adelaide, South Australia; Newell w. Johnson, MDSC, PhD, Population and Social Health Research Programme, Griffith Health Institute, Gold Coast, Queensland, Australia

Correspondence: Dr Steve Kisely, Level 4, Building 1, Princess Alexandra Hospital, 199 Ipswich Road, Woolloongabba, QLD 4102, Australia. Email: s.kisely@uq.edu.au

First received 14 Aug 2014, accepted 14 Mar 2015

\section{Funding}

This project was supported by the University of Queensland Winter Research programme.

\section{References}

1 Lawrence D, Hancock KJ, Kisely S. The gap in life expectancy from preventable physical illness in psychiatric patients in Western Australia: retrospective analysis of population based registers. BMJ 2013; 346: f2539.

2 Kisely S, Quek LH, Pais J, Lalloo R, Johnson NW, Lawrence D. Advanced dental disease in people with severe mental illness: systematic review and meta-analysis. Br J Psychiatry 2011; 199: 187-93. 
3 Romanos GE, Javed F, Romanos EB, Williams RC. Oro-facial manifestations in patients with eating disorders. Appetite 2012; 59: 499-504.

4 Hellstrom I. Oral complications in anorexia nervosa. Scand J Dent Res 1977; 85: 71-86.

5 Hurst PS, Lacey LH, Crisp AH. Teeth, vomiting and diet: a study of the dental characteristics of seventeen anorexia nervosa patients. Postgrad Med J 1977; 53: 298-305.

6 Milosevic A. Eating disorders and the dentist. Br Dental J 1999; 186: 109-13.

7 Frydrych AM, Davies GR, McDermott BM. Eating disorders and oral health: a review of the literature. Aust Dental J 2005; 50: 6-15.

8 Bretz W. Oral profiles of bulimic women: diagnosis and management. What is the evidence? J Evid Based Dent Pract 2002; 2: 267-72.

9 Lo Russo L, Campisi G, Di Fede O, Di Liberto C, Panzarella V, Lo Muzio L. Oral manifestations of eating disorders: a critical review. Oral Dis 2008; 14: 479-84.

10 Booth A, Clarke M, Dooley G, Ghersi D, Moher D, Petticrew M, et al. The nuts and bolts of PROSPERO: an international prospective register of systematic reviews. Syst Rev 2012; 1: 2.

11 Stroup DF, Berlin JA, Morton SC, Olkin I, Williamson GD, Rennie D, et al. Meta-analysis of observational studies in epidemiology: a proposal for reporting. Meta-analysis Of Observational Studies in Epidemiology (MOOSE) group. JAMA 2000; 283: 2008-12.

12 Metivier A, Bland K. Dental Anatomy: A Review. Dental Care.com, 2014 (http://www.dentalcare.com/media/en-US/education/ce421/ce421.pdf).

13 Slade GD, Spencer AJ, Roberts-Thomson KF. Australia's Dental Generations: The National Survey of Adult Oral Health 2004-06 (AlHW cat. no. DEN165). Australian Institute of Health and Welfare, 2007.

14 Dawes C. Physiological factors affecting salivary flow rate, oral sugar clearance, and the sensation of dry mouth in man. J Dent Res 1987; 66: 648-53.

15 Wells G, Shea B, O'Connell D, Peterson J, Welch V, Losos M, et al. The Newcastle-Ottawa Scale (NOS) for Assessing the Quality of Nonrandomised Studies in Meta-analyses. Available at: http://www.ohri.ca/ programs/clinical_epidemiology/oxford.asp.

16 Higgins JPT, Green S (eds). Cochrane Handbook for Systematic Reviews of Interventions version 5.0.2. Cochrane Collaboration, 2009.

17 Abramson JH. WINPEPI updated: computer programs for epidemiologists, and their teaching potential. Epidemiol Perspect Innov 2011; 8: 1.
18 Dynesen A, Bardow A, Petersson B, Nielsen LR, Nauntofte B. Salivary changes and dental erosion in bulimia nervosa. Oral Surg Oral Med Oral Pathol Oral Radiol Endod 2008; 106: 696-707.

19 Johansson AK, Norring C, Unell L, Johansson A. Eating disorders and ora health: a matched case-control study. Eur J Oral Sci 2012; 120: 61-8.

20 Ohrn R, Enzell K, Angmar-Mansson B. Oral status of 81 subjects with eating disorders. Eur J Oral Sci 1999; 107: 157-63.

21 Rytomaa I, Jarvinen $\mathrm{V}$, Kanerva $\mathrm{R}$, Heinonen OP. Bulimia and tooth erosion Acta Odontol Scand 1998; 56: 36-40.

22 Milosevic A, Slade PD. The orodental status of anorexics and bulimics. Br Dent J 1989; 167: 66-70.

23 Robb ND, Smith BG, Geidrys-Leeper E. The distribution of erosion in the dentitions of patients with eating disorders. Br Dent J 1995; 178: 171-5.

24 Liew VP, Frisken KW, Touyz SW, Beumont PJ, Williams H. Clinical and microbiological investigations of anorexia nervosa. Aust Dent J 1991; 36 435-41.

25 Emodi-Perlman A, Yoffe T, Rosenberg N, Eli I, Alter Z, Winocur E. Prevalence of psychologic, dental, and temporomandibular signs and symptoms among chronic eating disorders patients: a comparative control study. J Orofac Pain 2008; 22: 201-8.

26 Philipp E, Willershausen-Zonnchen B, Hamm G, Pirke KM. Oral and dental characteristics in bulimic and anorectic patients. Int J Eat Disord 1991; 10 423-31.

27 Altshuler BD, Dechow PC, Waller D, Hardy BW. An investigation of the oral pathologies occurring in bulimia nervosa. Int J Eat Disord 1990; 9: 191-9.

28 Smith BGN, Knight JK. An index for measuring the wear of teeth. Br Dent J 1984; 156: 435-8.

29 World Health Organization. Oral Health Survey - Basic Methods (4th edn). WHO, 1997.

30 Taylor C. Range Rule for Standard Deviation. How to Estimate the Standard Deviation. Available at: http://statistics.about.com/od/Descriptive-Statistics/ a/Range-Rule-For-Standard-Deviation.htm.

31 Roberts MW, Li SH. Oral findings in anorexia nervosa and bulimia nervosa: a study of 47 cases. J Am Dent Assoc 1987; 115: 407-10.

32 General Practice Queensland. Activate: Mind \& Body. General Practice Queensland, 2009 (http://www.gpqld.com.au/page/Programs/Mental_Health/ Improving_the_Physical_Health_of_People_with_a_Severe_Mental_Illness_ Project/). 